
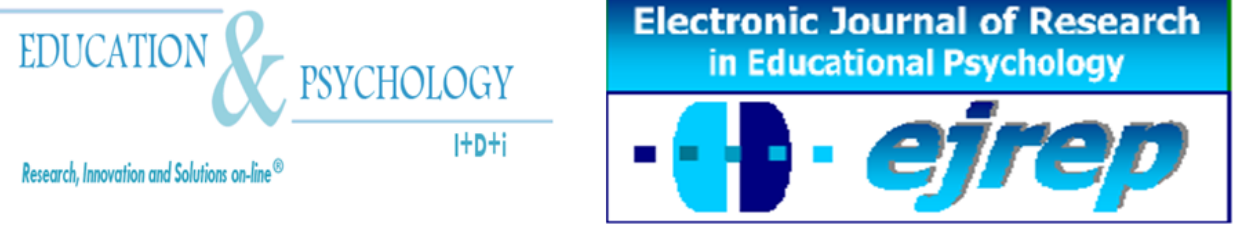

Editorial EOS

\title{
La revitalización de la Educación del Carácter en el ámbito psicoeducativo actual: Aportaciones desde las ciencias de la Prevención y la Psicología Positiva.
}

\section{Laura Vargas Villalobos ${ }^{1}, \mathrm{M}^{\mathrm{a}}$ Carmen González-Torres $^{2}$ \\ ${ }^{1}$ Responsabilidad Social, ITS Infocomunicación, San José \\ ${ }^{2}$ Departamento de Educación, Universidad de Navarra}

\section{${ }^{1}$ Costa Rica $/{ }^{2}$ España}

Correspondencia: $M^{a}$ Carmen González-Torres. Universidad de Navarra, Edificio de Biblotecas, Dpto de Educación. 31080. Pamplona, España. E-mail: mgonzalez@unav.es

(C) Education \& Psychology I+D+i and Editorial EOS (Spain) 


\title{
Resumen
}

¿Se está enriqueciendo la educación moral con las actuales aportaciones del ámbito de las Ciencias Psicosociales? Este artículo se dedica a estudiar el fenómeno del por qué de la revitalización de la noción de carácter y su educación ocurrido en Estados Unidos en la década de 1990. El lector podrá apreciar que dicho fenómeno está aportando un verdadero fortalecimiento y nuevas perspectivas a la educación moral. El objetivo de este trabajo es ofrecer una recopilación de las principales cuestiones relativas a lo que hemos denominado Movimiento de Revitalización del Carácter (MRC), abordando sus conceptos fundamentales, modelos más punteros y autores principales. Además, se busca resaltar su estrecha relación con algunas nuevas aportaciones desde el campo de la Prevención y la Psicología Positiva. Se vislumbra que, con sus avances actuales, el MRC será un punto de referencia para aquellos interesados por este tipo de educación.

Palabras Clave: Educación del Carácter, Positive Youth Development, Psicología Positiva, Resiliencia, Prevención.

Recibido: 30/06/09 Aceptación inicial: 07/07/09 Aceptación final: 22/09/09

\section{Revitalization of Character Education in the current Educa- tional Psychology arena: Contributions from the sciences of Prevention and Positive Psychology}

\begin{abstract}
Can moral education be enriched with recent contributions from the Social Sciences field? The purpose of this article is the analysis of the different factors that contributed to the resufacing of a "character" notion and to an increasing interest in its education in the United States during the decade of 1990. The reader will appreciate that this phenomena is adding a new reinforcement from different perspectives to moral education. The aim of this work is to offer a compilation of the main issues regarding what we have named the Character Revitalization Movement (MRC), approaching its cardinal concepts, most prominent models and main authors. We also want to highlight its close relation to some new valuable developments in the field of Prevention and Positive Psychology. We foresee that, with the recent advancements, the MRC will constitute a reference point to those interested in this type of education.
\end{abstract}

Keywords: Character Education, Positive Youth Development, Positive Psychology, Resilence, Prevention.

Received: 06/30/09 Initial Acceptance: 07/07/09 Final Acceptance: 09/22/09 


\section{Introducción}

"El carácter es el destino". Esta frase atribuida al filósofo Heráclito de Éfeso (544 a. C. - 484 a. C.) se ha incorporado a la sabiduría popular a través de los años, y su relevancia ejemplifica el recorrido milenario que ha tenido la noción de "carácter" en la historia humana. Este concepto -vinculado a la educación de virtudes- está adquiriendo una clara revitalización en el ámbito de la investigación y la práctica psicoeducativa en los EE UU que empieza a extenderse en otros países.

"Educación del carácter" se usa en los EE UU como un término general que agrupa iniciativas diversas, vinculadas con la educación en virtudes, educación en valores, educación para la ciudadanía, educación para la construcción personal, aprendizaje-servicio, educación emocional y educación de la afectividad. Aunque el concepto sigue todavía en construcción (Berkowitz y Bier, 2004), algunos rasgos parecen dar identidad a este tipo de educación: se utilizan las nociones de hábito y virtud (Lapsley y Narvaez, 2006), se busca que la educación moral sea integral -conocer el bien, amar el bien y hacer el bien- (Lickona, 1991), se cree que el buen carácter de los menores debe ser modelado y desarrollado intencionalmente por sus adultos significativos (Murphy, 2002).

Un botón de muestra de la centralidad de la educación del carácter en este país es la presencia que tuvo en el discurso de los dos candidatos que compitieron en las recientes elecciones presidenciales (2009). La reconocida asociación americana Character Education Partnership ha agradecido recientemente a B. Obama (presidente electo) sus palabras en pos de resaltar la urgencia "de restaurar la estatura moral de este pueblo (...)" (Character Education Partnership, 2009), aliándose así directamente a los objetivos de la Educación del Carácter. De igual manera el senador McCain (excandidato presidencial) resaltó la importancia que sigue teniendo la Educación del Carácter en esa sociedad, dedicando específicamente uno de sus libros a este tema (McCain y Salter, 2005). La importancia que Obama y McCain dan al "carácter" y su educación se hace eco de una corriente puntera nacida en este país en la década de los 90' del S. XX, y que hemos denominado Movimiento por la Revitalización del Carácter (en algunos momentos nos referiremos a él como Movimiento o en forma abreviada MRC). 
Nuestra intención es ofrecer una síntesis del estado de la cuestión sobre este movimiento y su gran a aportación a los retos actuales en la educación moral que sirva, a modo de “carta de navegación”, a los educadores e investigadores interesados por esta temática de la educación del carácter (en adelante, EC).

Durante siglos la noción de carácter y la importancia de su educación fue incuestionable pero por diversas causas -a las que haremos referencia-, sufrió un proceso de declive, a partir del cual llegó a ser un tópico prácticamente dejado de lado en las investigaciones psicológicas. Para explicar su revitalización resaltaremos la situación que hizo que los autores del MRC intentaran cambiar el rumbo de la educación moral tal como se venía impartiendo en los EE UU y por qué. Destacaremos su historia, sus principales propuestas y su capacidad para incorporar y robustecerse con algunas nuevas aportaciones desde las Ciencias de la Prevención -con su cambio paradigmático hacia el énfasis en los factores de protección, en las competencias y fortalezas de las personas para fomentar su desarrollo pleno (Positive Youth Development PYD) - y de la Psicología Positiva que camina en la misma línea.

\section{La revitalización de la educación del carácter como respuesta ante los retos actuales en la educación moral}

Actualmente hay un interés creciente por la formación personal y moral de los niños y adolescentes (Altarejos y Naval, 2000; De la Fuente, 2009; Iriarte, Alonso-Gancedo y Sobrino, 2006; López de Dicastillo Castillo, Iriarte, González-Torres, 2008; Nucci, 1997, 2005; Nucci y Narvaez, 2008; Trianes y Fernández, 2001). Sabemos que promover el desarrollo técnico e intelectual que se necesita hoy en día sin dotar a nuestros niños de la estructura moral para saber administrarlo sería, además de poco estratégico, irresponsable (Lickona, 1999, Lickona y Davidson, 2005).

La educación moral debe estar a la altura de los retos actuales. Muchos expertos en el trabajo con menores muestran una generalizada preocupación ante indicadores que señalan que algo anda mal con el estilo de vida de muchos niños y jóvenes Hablamos de crisis de valores, de violencia, de depresión infantil, de embarazos prematuros en adolescentes, de fracaso escolar, de excesivo hedonismo, de abuso de drogas o de falta de metas y de búsqueda de un sentido de la vida valioso (Damon, 1995, 2008; Elzo, 2006; Fernández-Berrocal y Aranda, 
2008; Gimeno Sacristán, 2005; González-Torres, 2001; 2003; Pérez y Cánovas 2002; Rodríguez, Muñoz Pérez y Sánchez, 2005; Urra, 2006).

Sin embargo, existen múltiples desacuerdos sobre cómo debe darse esta educación para que sea efectiva y les prepare para una vida saludable, feliz y solidaria. La educación moral de hoy en día tiene vicios profundos. Imperan en muchos centros educativos programas de diversa índole, que bajo la rúbrica “educación en valores” y otras, no está claro que doten a los niños y jóvenes de las herramientas necesarias para tomar buenas decisiones morales (Hoff, 2002; Lickona, 1991; Lickona y Davidson, 2005). También la educación en pro del fomento de la autoestima, contemplada como un foco de educación moral, ha sido muchas veces mal entendida, conduciendo a prácticas desenfocadas. El resultado de esto es un infundado temor a dañar el "self" del niño, lo cual se ha traducido en unas prácticas educativas y en una cultura con miedo a educar, a poner límites, a ejercer autoridad y en general a exigir algo que contradiga el ambivalente deseo del niño y del adolescente. Esto ha favorecido en los jóvenes el egocentrismo y el narcisismo, y paradójicamente la formación de una autoestima débil, "inflada” y frágil (Baumeister, Boden y Smart, 1996; Baumeister, Campbell, Krueger, y Vohs, 2005; Damon, 1995; González-Torres, 2001, 2003; Seligman, 1996; Smith y Elliot, 2001; Twenge, 2006; Twenge y Campbell, 2009; Urra, 2006).

Resulta urgente por tanto una reflexión sobre las posibilidades de la educación moral y muchas voces (desde la investigación, el ámbito práctico de la educación y de la prevención e intervención) han destacado la importancia de la EC en este sentido.

En la actualidad, el término "educación del carácter" es en los EE UU tan popular como controvertido (Lapsley y Narvaez, 2006). Desde el inicio de la década de los noventa hasta la fecha se ha producido un rápido desarrollo de bibliografía al respecto, de programas de intervención, de investigaciones teóricas y empíricas (Berkowitz y Bier, 2004, 2005a y b; Character Education Partnership, 1999; 2006; Lapsley y Narvaez, 2006; Murphy, 2001; Schaps, Battistich y Solomon, 1997). Narvaez (2005) ofrece cifras reveladoras: "al iniciar el siglo XXI, el número de escuelas que adoptan programas de EC en los Estados Unidos sigue en aumento: -de 51 Estados-, 47 reciben financiamiento para sus programas de EC, 14 Estados consideraban este tipo de educación como obligatoria". 
La EC no es una idea nueva. Los enfoques respecto a la educación del carácter se han dividido en dos visiones opuestas enraizadas en diferentes paradigmas filosóficos (Narvaez, 2008). El primero, se basa en el planteamiento clásico (en las aportaciones de Aristóteles) y se centra precisamente en el cultivo de la noción clásica "carácter" -ligado a la formación de hábitos y a la virtud-, mientras que el segundo, derivado del pensamiento de Rousseau, inició el proceso por el cuál la noción clásica de la EC dejó de ser el eje de la formación moral.

\section{La perspectiva moral aristotélica: La "Educación del Carácter"}

Aristóteles, en su Ética a Nicómaco ${ }^{1}$, entiende la virtud como una excelencia humana que permite a la persona realizarse al máximo y alcanzar la "vida buena". Según él, la vida virtuosa es el único camino para alcanzar la felicidad, y por tanto la plenitud humana (Bernal 1998, Naval, 2001; Palmour, 1986). Para Aristóteles, los hombres deben cultivar las virtudes (intelectuales y morales), cualidades que son objetivamente buenas. Una novedad de la contribución de Aristóteles a la teoría de la virtud es la apreciación de los tres componentes del desarrollo moral del individuo: intelectual, volitivo y conductual. El desarrollo de estos aspectos para la vivencia de las virtudes es el centro de la educación del carácter y se recoge en las definiciones de distintos líderes del MRC (Murphy, 2001). Así, por ejemplo Ryan señala que "virtud es la disposición a pensar, sentir y actuar de manera moralmente excelente (...) tener buen carácter significa ser una persona que tiene la capacidad de conocer lo bueno, amar lo bueno y hacer lo bueno" (Ryan y Bohlin, 1999, pág.5). La educación del carácter requiere fortalecer una disposición interna o facilidad (hábitos) para los buenos actos característicos de la virtud, que funciona como una espiral de crecimiento.

Según Aristóteles, en este proceso es de vital importancia el apoyo de la comunidad, pues aunque la persona está naturalmente constituida para vivir la virtud, siempre buscará evitarla si -como suele ocurrir- ésta trae consigo dolor o sufrimiento (Naval, 2001). Con la ayuda del profesor, los educandos aprenden a conocer, amar y practicar el buen actuar. El carácter es una segunda naturaleza, forjada voluntariamente por el mismo individuo, que le permite llevar una vida buena para sí mismo y para aquellos que le rodean (Lickona, 1999).

\footnotetext{
${ }^{1}$ Se hace referencia a la obra "Ética a Nicómaco" de Aristoteles, en la traducción hecha por Marias, 1994.
} 


\section{Declive de la noción clásica de "Educación del Carácter"}

La principal contrapropuesta a la filosofía aristotélica la hizo el francés J. Rousseau (S. XVIII). Este pensador propuso una idea romántica de persona inclinada naturalmente hacia el bien. Según él, para ayudar a los niños a vivir la virtud es necesario disminuir al máximo la influencia de los demás en ellos, pues los vicios se generan por un desorden interior causado durante la socialización. Rousseau proponía que se debe preparar al niño para la vida en sociedad dejándolo libre y sin influencias para que así desarrolle hábitos naturalmente. La persona será entonces dueña de sí misma, al escuchar solamente los deseos de su propia razón y voluntad. Diversas aproximaciones en educación moral tienen su base en esta idea rousseauniana, y presentan la autonomía como la meta principal de la educación moral (Bernal, 1998; Hoff, 2002).

Poco a poco, las ideas rousseaunianas fueron ganando aceptación y como consecuencia de esto, las nociones clásicas de carácter y virtud fueron dejadas de lado en el ámbito psicoeducativo. En este proceso influyeron propuestas y tendencias del pensamiento occidental del siglo XIX y XX como: la teoría evolucionista, la teoría de la relatividad, la adopción de metodologías experimentales en el ámbito de la psicología, las investigaciones sobre la naturaleza del carácter realizadas y difundidas por Hartshorne \& May, y las ideas personalistas que impulsó la psicología humanista (Lickona, 1999). Vale la pena destacar la importancia de estos tres últimos factores.

La utilización de metodologías experimentales en el ámbito de la psicología ocurrió como un intento de poner bases científicas a su quehacer. Los estudios psicológicos se centraron en las características humanas medibles y observables, desterrando constructos considerados vagos, generales y con fuertes connotaciones valorativas, como el de "carácter". Diversos autores indican que ha sido decisiva la influencia de la psicología en su corriente conductista en el oscurecimiento de la EC (Lickona, 1999, Peterson y Seligman, 2004).

El segundo factor que influyó en el declive de la EC fueron las investigaciones de $\mathrm{H}$. Harshorne \& M. May, bajo la supervisión de E. Thorndike, quienes publicaron sus resultados en el período comprendido entre 1928-1930, y que marcaron -tal vez sin pretenderlo- un antes y un después en la educación moral (Lapsley y Narvaez, 2006; Leming, 1997; Lickona, 1991; McClellan, 1992; Power, 1997). Los investigadores estaban interesados en esclarecer la 
estructura fundamental de lo que en la psicología y la educación se denominaba "carácter": buscaban determinar si se trataba de un conjunto de disposiciones estables naturales en la persona o más bien un grupo de capacidades adaptativas que dictaban una respuesta natural según cada situación (Leming, 2007).

Leming destaca dos conclusiones centrales de este estudio que cambiaron la aproximación de la psicología a la noción de carácter (Harshorne y May, 1930, Studies in the Nature of Character vol. 3, cit. en Leming, 1997). La primera básicamente negaba la existencia de patrones estables -como es el carácter- en el funcionamiento moral de los niños, utilizando como base para esta afirmación un experimento sobre conducta honesta/deshonesta en niños. Los autores afirman que "si la honestidad es una característica unificada del carácter, y todos los niños o la tienen o no la tienen, entonces, esperaríamos que los que la tienen sean honestos en todas las situaciones, y viceversa, que los deshonestos se comporten deshonestamente en toda situación. Lo que, de hecho, hemos observado es que la honestidad o deshonestidad en una situación está relacionada con la honestidad o deshonestidad en otra situación solamente en el grado que ambas situaciones tengan factores en común" (cit. en McClellan, 1992, p.61,). Hartshorne y May concluyeron entonces que no se podía demostrar que existieran comportamientos estables que pudieran identificarse como "carácter", y propusieron más bien que dependiendo de cada situación la persona se comportaba de manera diferente.

La segunda conclusión del estudio señalaba que los programas de EC que fueron evaluados no tenían un impacto efectivo en el comportamiento moral de los niños. En palabras de Hartshorne y May, "la simple exhortación de los profesores a actuar honestamente o la discusión de ideales o estándares de honestidad (...) no tiene necesariamente una relación con la conducta (...). Los métodos prevalecientes para inculcar ideales probablemente hacen muy poco bien e inclusive pueden hacer algún daño" (cit. en Leming, 1997, p.34). Con esto, se puso en tela de juicio la pertinencia de invertir recursos en la EC tal como se venía entendiendo hasta el momento.

Leming afirma que, en el ámbito de la investigación concretamente, los hallazgos de Hartshorne y May fueron un golpe mortal para la concepción de educación moral clásica y el descenso del interés por la noción de carácter. Para respaldar su afirmación cita dos investigaciones: la de Power, Higgins y Kohlberg en 1989, quienes encuentran que, entre los años de 1930-1940, la utilización del vocablo “carácter” disminuyó en su uso un 85\%. En segundo, la 
desarrollada por Stanhope en la que se indica que, mientras en el período de 1929-1939 fue posible encontrar 480 artículos sobre la educación del carácter, diez años después (el período de 1939-1948) solamente se encontraron 115 (una disminución del 76\%).

Finalmente, vale la pena señalar la influencia de Allport (1897- 1967), padre de la Psicología de la Personalidad y una importante influencia en el surgimiento de la Psicología Humanista. Peterson (2006a) destaca su influencia en la desaparición del término "carácter" del discurso académico relativo a la psicología de la personalidad, ya que este pensador propuso que el carácter era un término filosófico más que psicológico. Según Allport, los rasgos de personalidad que deberían estudiarse eran entidades con mayor grado de objetividad, desnudas de significado moral y orientadas a lograr una adaptación de la persona a su entorno. Por otra parte, los planteamientos acerca de la autorrealización de la psicología humanista también contribuyeron a cuestionar la idea tradicional de educar en unas virtudes y valores concretos.

Este era el contexto cuando la Teoría del Desarrollo Moral Cognitivo, propuesta por L. Kohlberg -fundamentada en las ideas Roussonianas, Kantianas y Piagetianas- dio el golpe de timón a la concepción de la educación moral vigente (en línea con la clásica noción de la EC) y se convirtió en el centro de estudio de la psicología y práctica moral. Según este autor (cit. en McClellan, 1992; Papalia et al., 2001; Peterson y Seligman, 2004), los niños progresan de manera más o menos ordenada a través de etapas de razonamiento moral que le dotan de una mayor autonomía para conocer y poner en práctica los principios morales fundamentales. El desarrollo del razonamiento moral es la esencia de la educación moral. El niño debe ser ayudado en este desarrollo para que avance por dichas etapas, y para ello Kohlberg propone ejercicios de razonamiento moral (con dilemas morales) y un modelo de centro educativo democrático en el que el niño toma parte en las decisiones para su buen funcionamiento. Según el autor, ambos elementos fortalecerán su capacidad de razonamiento moral, le orientarán hacia la justicia y le capacitarán para la vida democrática.

Propuestas de educación moral derivadas de la teoría del Desarrollo Moral Cognitivo y también de modelos como la Clarificación de Valores se pusieron en marcha en la mayoría de los centros de educación pública en los EE UU en los años 70 y 80 . Con el tiempo se demostró que no tuvieron los resultados esperados (Beningna, 1991; McClellan, 1992; Naval, 2001; Power, 1997; Quintana, 1995). Algunos autores (Hoff, 2002; Lickona y Davidson, 
2004) consideran que fueron las consecuencias de este tipo de educación lo que propició en gran medida el surgimiento del MRC en este país. El mero conocimiento racional del bien no lleva al estudiante al actuar moral, y por tanto era necesaria una educación que tomara en cuenta otros ámbitos de la persona, como los sentimientos, la motivación, los hábitos o las costumbres.

La educación del carácter en el contexto estadounidense y su resurgimiento a finales del siglo $X X$

En la última década del siglo XX se inicia en los EE UU un Movimiento (MRC) que busca reinsertar en el ámbito escolar y en la investigación la noción clásica de educación del carácter para vivir la virtud, y así dar respuesta a los problemas de los jóvenes que las otras propuestas de educación moral no habían conseguido. La revisión de la bibliografía publicada por los principales exponentes del MRC refleja su clara fundamentación en la filosofía griega (Bennigna, 1991; Bennigna y Wynne, 1998; Berkowitz, 1995, 1997, 1999, 2000, 2002; DeRoche y Williams, 1998; Lickona, 1991, 1997; Lickona y Davidson, 2005; Narvaez, 2002; Ryan, 1999; Ryan y Lickona, 1991; Wynne y Hess, 1991; Wynne y Ryan, 1997). Precisamente, una de sus características ha sido su intención de rescatar nociones antiguas -como la del carácter o la virtud- y a su vez encontrar nuevas metodologías para su educación, integrando ajustes o precisiones conceptuales dictadas por teorías más recientes.

Se pueden identificar tres factores que facilitaron que los educadores volvieran la mirada a los principios de la EC: a) la larga tradición a favor de esta educación, presente desde los inicios de la educación pública estadounidense; b) el particular proceso por el que se llevó a cabo su resurgimiento en este país en la década de los 90, donde fue vital el trabajo conjunto de distintas instituciones; c) los hallazgos de diversas investigaciones que contradecían las conclusiones del estudio de Harshrone y May y la integración de teorías que, recogiendo las aportaciones de la educación moral según los principios del Desarrollo Moral Cognitivo, rescataban el papel de las virtudes y el carácter en la vida humana (Narvaez, 2008).

En alusión al primer punto, autores como Cunningham, 2005; Damon, 2002; Devine, Seuk, Wilson, 2000; McClellan, 1992, 1999 y Nash, 1997 afirman que desde sus inicios, la educación pública en EE UU se entendió como una extensión de la "escuela dominical", de ahí el claro carácter moral-religioso que tuvo. Las repetidas olas de inmigración activas hasta 
la actualidad han hecho de esta nación un "crisol" cultural. Para responder a los retos de esta integración, el gobierno tomó la decisión de valerse de la educación pública para uniformar los valores de la naciente república. Se optó por adoptar los principios cristianos comunes a las denominaciones protestantes presentes en el país, y por esta razón se siguieron los planteamientos clásicos de la EC. Esto ha permitido que la sociedad estadounidense estuviera familiarizada con ellos.

El constante interés por la educación moral en los EE UU ha promovido la investigación continua y la puesta en marcha de metodologías en pos de una educación moral más efectiva. Así, de la misma manera que este interés facilitó la rápida adopción del modelo del Desarrollo Moral Cognitivo, también ha facilitado la vuelta a la EC y el desarrollo de las propuestas del MRC.

Por otra parte, un aspecto particular ha sido el proceso por el cuál tomó forma el MRC en la década de los 90. En este fue vital el trabajo conjunto entre el gobierno, ONGs, centros de investigación y centros educativos. Su trabajo en conjunto promovió una rápida difusión por todos los EE UU. Recopilando las aportaciones de Cunningham (2005), Devine et al., 2000; De Roche y Williams (1998), Hoff (2002), McClellan (1999); Williams, Yanchar, Jensen y Cherryl (2003), se puede hacer un listado de los acontecimientos que hicieron posible la introducción de los principios de EC en la política educativa pública estadounidense a principios de la década de los años 90 del S.XX. Entre estos se pueden señalar por orden cronológico:

1. En 1992 la organización Josephson Institute of Ethics reunió en Aspen, Colorado a un grupo de 30 líderes nacionales (entre ellos profesores, líderes juveniles, políticos y expertos en ética) para reflexionar en torno a la EC y potenciar el trabajo conjunto (Character Counts!, 2007a y b). La Aspen Declaration on Character Education, se convirtió en un hito que marcó los inicios del MRC (Hoff, 2002). Fue trascendental la afirmación de que existía una lista de valores centrales -core values-comunes a toda la sociedad y que, por tanto, podían ser la base de la educación moral en todo el país. Se buscaba así superar el relativismo ético (Lapsley y Narvaez, 2006) que imperaba en la educación moral basada en el Desarrollo Moral Cognitivo. Los participantes de la reunión que dio a luz la Aspen Declaration se comprometieron a impulsar una educación moral que promoviera estos valores centrales. 
2. A la luz de esta experiencia, en 1993 surgieron dos organizaciones sin ánimo de lucro que desde entonces promueven la EC en todo el país. La primera es Character Counts! Coalition, y la segunda es la Character Education Partnership (CEP)

3. Desde sus inicios, el MRC ha contado con un importante apoyo del gobierno, independientemente de la ideología política de su presidente o congreso. Varios hechos demuestran este apoyo, resaltamos solamente dos: a) desde el año 1995 el gobierno federal ha ofrecido becas para ayudar a los estados a poner en marcha programas para la EC; en ese mismo año, once Estados se beneficiaron de subvenciones especiales que hacían posible el planeamiento de metodologías que promovieran la $\mathrm{EC}$ en todos sus colegios públicos; y b) en el 2002 se decidió que el tema de la conferencia anual de la Casa Blanca fuera "Carácter y Comunidad", reuniendo a los principales autores del MCR (Damon, Narvaez, Schaps, Walker entre otros).

4. En 1996 ve la luz el Character Education Manifesto, que pretendía unificar los principios centrales de la EC. Fue el primer documento de este tipo que planteó una definición común de la noción de carácter, además de proponer siete principios guía para las reformas educativas que se pretendían implantar en los programas educativos (Center for the Advancement of Ethics and Character, 2006). Al Manifiesto se suscribieron los gobernadores de ocho estados, un hecho que le dio una visibilidad importante.

5. Finalmente, vale la pena resaltar los esfuerzos de diversos investigadores por proponer modelos propios que delimitaran el mejor camino para el MRC (Lapsley y Narvaez, 2006; Lickona y Davidson, 2005) y ofrecieran criterios de análisis para identificar las "buenas prácticas” (Berkowitz y Bier, 2005a; CEP, 1999, 2006). Así, la expansión del Movimiento ha sido potenciada gracias a programas de reconocimiento anual a las mejores iniciativas de EC puestas en marcha en muchos centros educativos del país.

6. En estos últimos años se han desarrollado en los EE UU otras perspectivas desde diversos ámbitos de las ciencias y práctica psico-social con objetivos comunes a la EC que, por la solidez teórica y empírica de sus conclusiones han hecho que ésta cobre importancia en el ámbito del trabajo preventivo con niños y jóvenes constituyendo un importante respaldo para el MRC (Battistich, 2005; Lapsley y Narvaez, 2006).

\section{Aportaciones desde las Ciencias de la Prevención y la Psicología Positiva}

En las propuestas del MRC, es posible diferenciar dos etapas en su desarrollo. La primera, denominada "perspectiva tradicional de la EC", abarca la década de los 90 y se caracte- 
riza por ser una etapa de concienciación y difusión de los beneficios de este tipo de educación (Bennigna y Wynne, 1998; Lickona, 1991, 2004a, b; Ryan y Bohlin, 1999; Wynne y Hess, 1991; Wynne y Ryan, 1997); adolece, sin embargo, de un desarrollo teórico consistente y de estrategias de medición y validación de los resultados de sus modelos y propuestas. Estas carencias intentan subsanarse en una segunda etapa, donde se desarrollan modelos que, fundamentados en las aportaciones de la Psicología y en los avances científicos en las áreas en las que se apoya el ámbito de la Prevención, ofrecen conceptos y metodologías que facilitan la educación en virtudes (Berkowitz, 2000, 2002; Berkowitz y Bier, 2004, 2005a y b; Catalano, Hawkons y Toumbouro, 2008; Lickona y Davidson, 2005; Narvaez, 2005).

Cambios en el área de la Prevención: Del déficit y los factores de riesgo hacia el interés por las fortalezas y los factores de protección

Paralelo al MRC se produjo en la década de los 90' un cambio de paradigma o perspectiva en el trabajo preventivo con niños y jóvenes que se recoge bajo lo que se ha denominado Positive Youth Development (en adelante PYD) apoyado también por la naciente Psicología Positiva. La revisión bibliográfica revela que tanto los promotores de esta orientación como los autores del Movimiento han identificado una estrecha convergencia entre sus planteamientos que puede ser mutuamente beneficiosa (Battistich 2005; Berkowitz, 2000; Berkowitz, Sherblom, Bier, Battistich, 2005; Lapsley y Narvaez, 2006; Narvaez, 2005; Park, 2004; Schaps, Battistich, y Solomon, 1997).

¿En qué consiste el cambio paradigmático al que nos referimos? Se trata de un movimiento a favor de una perspectiva positiva para enfocar los servicios, programas y las políticas preventivas diseñadas para atender los problemas y el desarrollo de los jóvenes. Bajo el PYD se recogen los hallazgos recientes de distintas ciencias de la conducta sobre el enorme potencial del desarrollo humano incluso en situaciones muy adversas y ha aunado a científicos, políticos y trabajadores del ámbito práctico de la prevención y de otros ámbitos científicos, entre ellos, los que se ocupan de la EC (Narvaez, 2008).

Presentar en este apartado los elementos comunes que resuman el trabajo realizado desde esta perspectiva es una tarea difícil. Benson y Saito (2001) llegan a afirmar que si se les pidiera a 10 personas elaborar una reseña que sintetice lo que se conoce actualmente sobre el PYD emergerían 10 reseñas muy diferentes entre sí y el cruce de referencias sería mínimo. 
Intentaremos, sin embargo, ofrecer una breve síntesis de las ideas centrales en esta iniciativa y de su relación con el MRC. Hay que destacar que un intento de ofrecer una definición operacional de juventud positiva lo realizaron ya en 1997 Catalano et al. (2008).

K. Pittman -autora puntera en el lanzamiento del PYD- lo describe como un movimiento centrado en que en el campo de la prevención se ponga el acento en el desarrollo de las fortalezas y competencias de los niños y jóvenes y no sólo en la reducción de problemas (Pittman, et al., 2001). Este movimiento (Benson y Pittman, 2001; Berkowitz et al., 2005c; Catalano et al., 2004; Clary y Rhodas, 2006; Damon, 1995, 2002; Pitman, Irby y Ferber, 1996; Pittman et al., 2001; Roth y Brooks-Gunn, 2003; Small y Memmo, 2004) buscaba que la prevención no sólo se orientara a proteger a determinados niños y jóvenes contra las conductas de riesgo más frecuentes y con programas muy específicos centrados en determinadas problemáticas (drogadicción, violencia, etc.), sino que comenzaba a vislumbrarse que para hacer prevención era necesario invertir recursos en toda la población, sea esta catalogada como sana, enferma o en riesgo (Damon, 2002). Debía también ocuparse de fortalecer una amplia gama de recursos personales capaces de ayudarles no sólo a superar esos riesgos o cualquier otro presente en la vida diaria sino a tener unas vidas más plenas y felices. Se buscaba superar la extendida estereotipación del desarrollo adolescente y juvenil como un período lleno de peligros, en el que los menores eran vistos "como problemas" que deben de ser enderezados a tiempo antes de que se hagan daño a sí mismos o a los demás. También había que empezar a ver a los jóvenes más "como recursos" que como "problemas" y adoptar una perspectiva multicausal y ecológica (implicar a las escuelas, familia y comunidad más amplia en el desarrollo positivo de los jóvenes).

Pittman et al. (2001, p.4) ofrecen un ejemplo que se considera muy útil para ilustrar lo expuesto: en el supuesto que se haga la presentación de un joven para una entrevista de trabajo de la siguiente forma "aquí está Juan. No consume drogas. No pertenece a ninguna pandilla. Hasta el momento, no ha presentado absentismo. No es un padre adolescente. Por favor, contrátenlo" lógicamente, el empleador dirá algo similar a: "Eso es una buena noticia, sobretodo en estos tiempos pero... ¿qué sabe hacer Juan?” Así, el lema de los promotores de este cambio paradigmático, que se ha denominado PYD, fue "estar libre de problemas no significa estar bien preparado". Se considera que esta frase resume el meollo del problema: una percepción bastante limitada sobre la juventud, sus posibilidades y sus necesidades. La 
pregunta “¿qué pueden hacer los jóvenes?” es el foco central del PYD: las capacidades de cada joven y los mecanismos para fortalecerlas.

Dos líneas de investigación han contribuido notablemente al desarrollo del PYD: a) las investigaciones sobre la resiliencia y b) el estudio de los llamados Developmental Assets (en adelante DA) (Masten y Coatsworth, 1998).

Con las teorías sobre el desarrollo "resiliente" se intenta explicar un hecho "misterioso" con el que se topaban científicos y trabajadores del ámbito de la salud con cierta frecuencia: ciertos individuos expuestos durante su infancia a graves riesgos (e.g. pobreza, alta posibilidad de heredar enfermedades genéticas, violencia, desintegración familiar y/o secuelas de guerra o catástrofes naturales) aparentemente eran capaces de salir adelante sin ayuda, desafiando los fatales resultados esperados. Se entiende por resiliencia esta competencia manifestada a pesar de la exposición a estresores significativos (Rolf y Glantz, 1999). Dos aspectos son necesarios para que se de la resiliencia: la exposición a un riesgo o presión importante y la posterior adaptación positiva al entorno (Masten, 1999, 2001; Masten y Reed, 2002). La capacidad resiliente significa para la persona una protección al posible riesgo y al mismo tiempo, un recurso para llevar una vida sana en su entorno específico.

Una de las aportaciones más significativas en este campo fue la investigación intercultural de E. Werner (1996), realizada con niños inmersos en contextos de alto riesgo en Hawai y Estados Unidos continental. En su estudio longitudinal, siguió el proceso de desarrollo de casi 700 niños en situación de pobreza y marginalidad, registrando los factores de riesgo presentes en el contexto en que nacían y siguiendo su trayectoria personal durante 40. Entre sus conclusiones destaca que la resiliencia ocurre con más frecuencia de lo pensado y es parte de lo que Masten (2001) denomina una "magia de la vida ordinaria". Del estudio se resaltó que solamente 1 de cada 6 niños expuestos a por lo menos un factor de alto riesgo mostraban a los 32 ó 40 años alguna conducta desadaptativa. Existen en la actualidad diversos estudios que apoyan esta aseveración: lejos de ser un fenómeno extraordinario, la resiliencia parece ser una característica común en los seres humanos antes subestimada por los científicos sociales (Bonnano 2004; Brown, D’Emidio, Bernard, 2001; Glantz y Johnson, 1999; Henderson, Benard, Sharp-Light, 1999; Masten y Reed, 2002). La resiliencia no es entonces una cualidad o un grupo de cualidades especiales de unos "niños invencibles". 
El estudio de Werner señaló que era posible identificar algunos elementos en común del contexto y de la personalidad de los individuos resilientes, características que fueron llamadas "factores protectores". Ese término hace referencia a las características de un grupo de individuos o su situación particular, que son medibles y pueden predecir resultados positivos ante un contexto de riesgo o adversidad (según Masten y Reed, 2002). Son como una especie de escudo que conserva la estructura interna de la persona, impidiendo que el estrés al que está expuesta deteriore su salud física o mental.

Diferentes autores han propuesto listados diversos que enumeran los factores protectores más efectivos para generar una estructura resiliente (Bernard, 2004; Masten y Reed, 2002; Morrison et al., 2006; Munist et al., 1998). La propuesta de Bernard (2004) que ha tenido enorme influencia en los sectores de orientación práctica del campo del desarrollo juvenil, clasifica estos factores en dos grandes grupos: a) factores protectores internos o fortalezas que son características propias del individuo que le hacen capaz de responder positivamente al estrés y, b) factores protectores externos o factores ambientales: características del ambiente que rodea al niño y que favorecen el desarrollo de los factores protectores internos. El primer grupo de factores puede verse en la tabla1:

Tabla 1. Factores protectores internos Tomado de Bernard, 2004, p. 14.

\begin{tabular}{|c|c|c|c|}
\hline Competencia social & $\begin{array}{c}\text { Resolución de } \\
\text { Problemas }\end{array}$ & Autonomía & Sentido de la vida \\
\hline Receptividad & Planificación & Identidad positiva & $\begin{array}{c}\text { Orientación a me- } \\
\text { tas, motivación } \\
\text { hacia el logro, aspi- } \\
\text { raciones educativas }\end{array}$ \\
\hline Comunicación & Flexibilidad & $\begin{array}{c}\text { Locus de control interno e } \\
\text { iniciativa }\end{array}$ & $\begin{array}{c}\text { Intereses especiales } \\
\text { o pasatiempos, crea- } \\
\text { tividad, imaginación }\end{array}$ \\
\hline Empatía/caring & Ingenio & $\begin{array}{c}\text { Autoeficacia y maestría } \\
\text { (mastery) }\end{array}$ & Optimismo \\
\hline Compasión, altruismo & $\begin{array}{c}\text { Pensamiento críti- } \\
\text { co e introspección }\end{array}$ & $\begin{array}{c}\text { Desapego adaptativo y } \\
\text { resistencia pasiva }\end{array}$ & Esperanza \\
\hline & & $\begin{array}{c}\text { Atención /conciencia plena } \\
\text { (mindfullness })\end{array}$ & $\begin{array}{c}\text { Fe, espiritualidad, } \\
\text { sentido de la vida }\end{array}$ \\
\hline & Sentido del humor & \\
\hline
\end{tabular}


Bernard incluye dentro de los factores protectores externos: a) el caring/ soporte afectivo, b) las altas expectativas en el ambiente que rodea al niño y c) las posibilidades para la partipación/contribución en la comunidad circundante.

Tomando como base estas investigaciones, se desarrolla a mediados de los 90 el trabajo sobre los Developmental Assets (DA) de P. Benson y sus colegas del Search Institute en Minnesota (Benson et al., 1999; Benson y Pittman, 2001; Benson y Saito, 2001). Este Instituto buscaba estudiar los talentos, las energías, las fortalezas y los intereses constructivos más importantes que ayudan a los jóvenes a tener un desarrollo saludable en todos los órdenes. Su inquietud surge en el marco de la preocupación generalizada por los índices negativos de “conducta saludable" en la niñez y adolescencia en EE UU en esa década. A partir de la revisión de múltiples investigaciones al respecto, el grupo hace su propuesta con la intención de brindar un marco que facilitara la construcción de bases sólidas que permitiera la promoción del bienestar integral de los jóvenes y capacitarlos para hacer frente a sus problemas. Su propuesta se ha convertido en una de las más conocidas y influyentes en el marco del PYD (Small y Memmo, 2004).

En respuesta al lema del PYD "libre de problemas no significa bien preparado" (Pittman, 2001; Roth y Brooks-Gunn, 2003; Small y Memmo, 2004), los promotores de este enfoque buscan identificar qué significa "el estar bien preparado" y cómo ayudar a los jóvenes a estarlo. La clasificación que propone el Search Institute incluye 40 características clasificadas en 8 subgrupos. Por un lado están los external assets (recursos externos) y por el otro los internal assets (recursos internos), cada uno incluye veinte características positivas organizadas en cuatro categorías (ver figura 1).

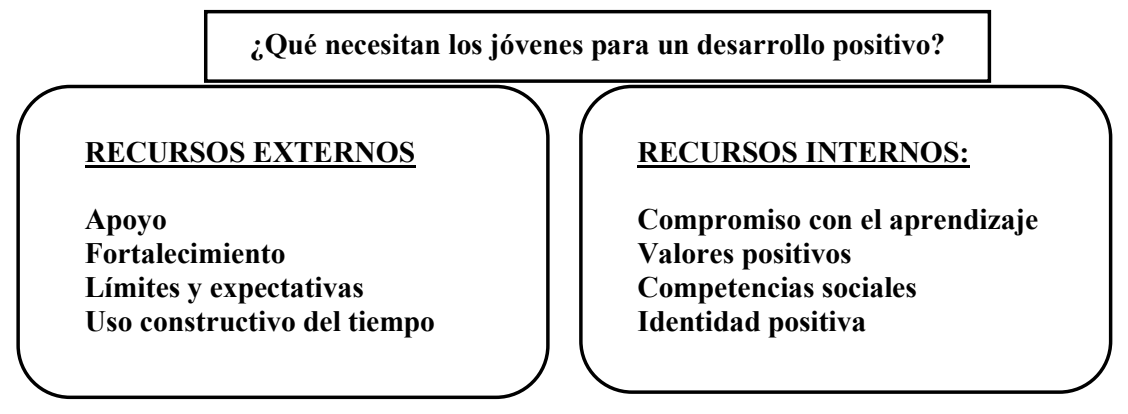

Figura 1. Recursos externos e internos Inspirado en Benson et al., 1999. 
Actualmente, el Search Institute (Search Institute, 2007) está validando modelos sistemáticos de assets para otros grupos etarios (niños de edad preescolar, niños de escuela primaria) y su clasificación de DA se ha empleado en proyectos e investigaciones educativas entre diversas instituciones.

Ha transcurrido poco más de una década y a pesar del corto tiempo hay una gran cantidad de personas e instituciones que han puesto en marcha programas a favor del desarrollo juvenil según los principios del PYD con resultados interesantes (Battistich, 2005; Catalano y cols., 2004, 2008; Roth y Brooks-Gunn, 2003) y que son coincidentes con muchas de las ideas de los proponentes de la EC. Pero la práctica va por delante de la teoría y quedan importantes retos por superar (Benson y Saito, 2001; Berkowitz et al., 2005, Catalano et al., 2008). La llamada "Psicología Positiva", con su estudio sobre las fortalezas del carácter y las virtudes, está contribuyendo sustancialmente en este intento.

\section{La Psicología Positiva y las fortalezas de carácter}

El cambio paradigmático en las Ciencias de la Prevención también se aprecia en la orientación de la Psicología que se conoce como "Psicología Positiva", que nace de la mano de Seligman en 1998, y que ha experimentado un gran desarrollo e interés en muy pocos años (Chafouleas y Bray, 2003; Keyes y Haidf, 2003; Linley y Stephen, 2004; Linley, Stephen, Harrington y Wood, 2006; Lopez, Snyder y Rasmusen, 2003; Maddi, 2006; Park et al., 2006; Seligman, Steen, Park, Peterson, 2005; Sheldon y King, 2001; Roberts, Brown, Johnson, Reinke, 2002; Vera, 2006).

Sus principales promotores la describen como una ciencia que busca estudiar: a) las experiencias subjetivas positivas (e.g. felicidad, satisfacción vital, emociones positivas), b) los rasgos individuales positivos (e.g. carácter, valores, resiliencia) y c) las instituciones positivas (e.g. familia, centros educativos, comunidad), con el objetivo de mejorar la calidad de vida de las personas y prevenir las patologías mentales (Seligman y Csikszentmihalyi, 2000).

Señalan que este enfoque pretende llamar la atención sobre la importancia de que la Psicología y las demás Ciencias Sociales "pongan la lente" en aquellas características positivas que hacen la vida satisfactoria y que son comunes en la vida de la mayoría de las personas, antes que en lo que va mal en ellas (las patologías o déficits de cada uno). Según sus 
planteamientos, lo importante no es sólo la ausencia de la enfermedad sino el desarrollo de las emociones positivas, las fortalezas personales y las instituciones que promuevan la felicidad, la salud y la vinculación social. Estos autores manifiestan su expectativa de que en este siglo XXI ésta se desarrolle como una ciencia y profesión que llegue a construir -y no sólo comprender- los factores que permiten a los individuos, comunidades y sociedades florecer y llevar vidas satisfactorias y plenas.

La Psicología Positiva ha rescatado la atención científica sobre el carácter como queda resaltado en la siguiente afirmación de Seligman: "toda ciencia que no utilice el carácter como idea básica (...) nunca será aceptada como ilustración útil de la afectividad humana. Por consiguiente, considero que ha llegado el momento para resucitar el carácter como concepto central del estudio cientifico del comportamiento humano.” (Seligman, 2003, p.193).

Una de las principales aportaciones de esta corriente de la mano de Peterson, Seligman, Park y otros ha sido el desarrollo del proyecto The Values in Action (VIA) Classification of Strengths que buscaba identificar las virtudes y fortalezas de carácter que era necesario desarrollar en todo ser humano para asegurar una "vida buena" en el sentido aristotélico y que además actuarían como una barrera protectora contra los riesgos o problemas psicológicos, por lo que el desarrollo de las mismas sería claramente una estrategia preventiva (Park, 2004; Park y Peterson, 2009; Peterson y Seligman, 2004; Seligman et al., 2005; Steen, Kachorek, Peterson, 2003).

Según Peterson (2006a), el proyecto surgió a la luz de las dificultades que existían en el seno del PYD para lograr una definición clara de "desarrollo óptimo" con la que establecer estrategias de común acuerdo que guiaran las intervenciones eficaces para lograrlo. Sin conceptos ni mediciones comunes, resultaba imposible determinar los ingredientes esenciales que deberían guiar el diseño de las intervenciones desde la prometedora perspectiva del PYD. El presidente de la Mayerson Foundation -N. Mayerson- estaba interesado en colaborar en proyectos que promovieran el PYD y preguntó a Seligman si desde la Psicología Positiva podría surgir alguna aportación al respecto (VIA, 2007). Con el interrogante “¿cómo podemos ayudar a los jóvenes a realizar su máximo potencial?" (Peterson y Seligman, 2004) fundan en conjunto, en el año 2000, el Instituto VIA, un proyecto que unió ambas perspectivas -el PYD y la Psicología Positiva-. 
La investigación en la que se embarcaron Peterson, Seligman y colaboradores proporcionó el punto de partida para el estudio sistemático del carácter y tuvo como resultado una clasificación de 6 virtudes nucleares, universales, que hacen posible "la vida buena", contribuyendo al desarrollo óptimo de la persona. También definieron 24 "fortalezas de carácter" los ingredientes psicológicos (procesos o mecanismos) que definen las virtudes y que son las rutas distinguibles para manifestarlas- (véase La Clasificación VIA de Fortalezas de Carácter en la tabla 2).

Además crearon un instrumento de medición para adultos que evalúa el grado de presencia de esas virtudes básicas y "fortalezas de carácter", denominado Values in Action Inventory of Strenghts (VIA-IS). Peterson y Seligman publicaron su clasificación e instrumento, en el 2004, en el libro Character Strengths and Virtues: A Handbook and Classification, editada por Oxford Press con el apoyo de la American Psychological Asociation. Esta obra ha sido considerada como el primer gran proyecto desarrollado deliberadamente desde la Psicología Positiva (Peterson, 2006b). Por otro lado, como reconocen Park y Peterson (2009 p.3) "el VIA Project está proporcionando un vocabulario legitimado para el debate psicológicamente informado acerca de las cualidades de los individuos que los hacen valiosos moralmente".

Vale la pena resaltar también la aportación de Park (2004) sobre la relación entre ésta, el PYD y la EC expuestas en su artículo Character Strenghts and Positive Youth Development. Así afirma que desde el marco conceptual de la Psicología Positiva, es posible encontrar un esquema comprensivo que facilite la promoción del desarrollo positivo en niños y jóvenes que se logra a través de la EC. Park le da valor al trabajo realizado por el MRC en pos del rescate de los términos de virtud y carácter, resaltando el respaldo científico que está teniendo con las aportaciones del PYD y la Psicología Positiva. Además, es la autora de una versión del instrumento para jóvenes (10-17 años), denominado VIA Youth Survey.

A continuación presentamos brevemente algunos de los más recientes avances del MRC que integran las aportaciones señaladas. 
Tabla 2. Clasificación VIA de Fortalezas de Carácter, inspirado en Vera, 2008

\begin{tabular}{|c|c|}
\hline Virtud & Fortaleza de Carácter \\
\hline Sabiduría y conocimiento & $\begin{array}{c}\text { Curiosidad, interés por el mundo } \\
\text { Amor por el conocimiento y el aprendizaje } \\
\text { Juicio, pensamiento crítico, mentalidad abierta } \\
\text { Ingenio, originalidad, inteligencia práctica } \\
\text { Perspectiva }\end{array}$ \\
\hline Coraje & $\begin{array}{c}\text { Valentía } \\
\text { Humanidad }\end{array}$ \\
\hline Trascendencia & $\begin{array}{c}\text { Perseverancia y diligencia } \\
\text { Integridad, honestidad, autenticidad } \\
\text { Vitalidad y pasión por las cosas }\end{array}$ \\
\hline Justicia & $\begin{array}{c}\text { Amor, apego, capacidad de amar y ser amado } \\
\text { Simpatía, amabilidad, generosidad } \\
\text { Inteligencia emocional, personal y social }\end{array}$ \\
& $\begin{array}{c}\text { Esperanza, optimismo, proyección hacia el futuro } \\
\text { Sentido del humor } \\
\text { Ciudadanía, civismo, lealtad, trabajo en equipo } \\
\text { Sentido de la justicia, equidad } \\
\text { Liderazgo }\end{array}$ \\
\hline
\end{tabular}

\section{Nuevos avances del MRC: Aportaciones de Berkowitz, Lickona y Narváez}

Al hacer un análisis del camino recorrido por el MRC, Berkowitz y Bier (2005 a y b) afirman que nos topamos con el eterno problema del "vaso medio vacío o vaso medio lleno". ¿Debemos poner la mirada en la gran cantidad de programas que hasta la fecha se han implementado para la EC, o más bien en la falta de precisión conceptual y de rigor científico?

Al iniciar el siglo XXI termina una primera etapa del MRC. Durante ella hubo grandes avances, entre ellos el apoyo de la sociedad estadounidense para los objetivos de la EC y el establecimiento de los fundamentos teóricos del Movimiento. Con el cambio de milenio se 
inicia una segunda etapa, donde la mirada se centra en perfilar más profundamente el corpus teórico acerca del carácter y su educación avanzando en la incorporación de las aportaciones de otras disciplinas y en la verificación empírica de propuestas de formación del carácter. En el 2006, Lapsley y Narvaez publican un trascendental capítulo titulado "Educación del Carácter" en el Handbook of Child Psychology (volumen coordinado por W. Damon), una obra de renombrado prestigio mundial que es punto de referencia en el campo Psicología del Desarrollo y de la Educación, lo cual pone de relieve el interés actual de esta temática. En esa revisión se destacan las aportaciones de Berkowitz, Lickona y Narvaez. Estos autores también han colaborado en el reciente libro titulado Handbook of Moral and Character Education coordinado por Nucci y Narvaez (2008).

Sus aportaciones tienen en común: a) la búsqueda de una mayor precisión terminológica, b) un mayor soporte en teorías psicopedagógicas actuales, y c) experiencias concretas de aplicación que han fortalecido los modelos teóricos (en el caso de Lickona y Narvaez).

Martin Berkowitz y su modelo de Anatomía Moral:

Berkowitz es especialista en Psicología del Desarrollo, profesor en la Universidad de Missouri (Saint Louis) y dirige el McDonnell Leadership Academy in Character Education, un centro centrado en el trabajo con directores de centros educativos para la promoción de la EC. Se pueden destacar de él tres aportaciones principales:

a) Un modelo que ilustra la relación del carácter con la estructura moral humana propuesto en la década de los 90`s. Berkowitz $(1997,1998,1999)$ con él pretende identificar los componentes de la moralidad humana para así precisar cuál debe ser el abordaje de la EC. El principio clave del que parte es que la EC (y la Psicología Moral) ha simplificado su aproximación a la moralidad humana al entenderla como un constructo relativamente homogéneo. Considera que se debe entender más bien como un complejo grupo de constructos de diverso tipo que engloban a la totalidad de la persona moral. Para comprender el funcionamiento moral de la persona se plantea el siguiente interrogante: "si tuviésemos que diseccionar a la persona moral y categorizar los diversos órganos morales que encierra la moralidad humana, ¿qué incluiríamos en la lista?” (Berkowitz, 1995, p.3). Él propone siete componentes de esta anatomía moral humana: conducta moral, carácter moral, valores morales, razonamiento moral, emociones morales, ejemplaridad moral y funcionamiento moral efectivo. Señala que de- 
ben plantearse estrategias que respondan a la naturaleza de cada uno de los componentes de la “anatomía moral”, para facilitar así el desarrollo positivo integral de la persona. Según Berkowitz (1997) el carácter es una de las dimensiones de la moralidad humana, y lo define como un grupo de características psicológicas del individuo que afectan a su habilidad e inclinación para actuar moralmente.

b) El abogar por un trabajo conjunto entre las Ciencias de la Prevención Positiva y el MRC, pues comparten objetivos, términos y población meta. Fue uno de los primeros en resaltar la importancia de que en las propuestas del MRC se integraran diferentes teorías y metodologías de campos afines para responder así mejor a la complejidad y diversidad del funcionamiento moral. En sus escritos destaca la importancia que se da al papel de la EC dentro del marco del PYD por su función preventiva (Berkowitz, 2000; Berkowitz et al., 2005).

c) La valoración crítica de los avances y puntos de mejora de la Educación del Carácter durante su $1^{\mathrm{a}}$ y $2^{\mathrm{a}}$ etapa. En un estudio titulado What works on character education (Berkowitz y Bier, 2005a y b) y financiado conjuntamente por la Character Education Partnership y la John Templeton Foundation, hace una revisión de las iniciativas que se declaraban a sí mismas como "educadoras del carácter". Su objetivo fue identificar las características de los programas que tenían buenos resultados comprobados empíricamente. La síntesis de "mejores prácticas" y recomendaciones que ofrece ha servido de base firme para el crecimiento del MRC. En un artículo posterior, (Berkowitz et al., 2008) tratan de identificar cuál es el sustrato teórico común del MRC, y cuáles deben ser las líneas futuras de investigación.

Narvaez (2005) ha resaltado que el modelo de Berkowitz es un puntal importante en el rescate de la EC como ámbito de estudio dentro de la Psicología de la Educación. Considera que su mayor aportación ha sido establecer la relación del carácter con las nociones de personalidad e identidad. En cuanto a sus críticas destaca la falta de precisión de las definiciones de los componentes de su modelo y la carencia de un respaldo empírico para su propuesta.

\section{Thomas Lickona y el modelo Escuelas Secundarias Buenas e Inteligentes}

En el año 2005, Lickona -professor de la New York State University y fundador del Center for the 4th and 5th R's: Respect and Responsability (2006)- saca a la luz, en colaboración con M. Davidson, un trabajo titulado Smart and Good High Schools: Integrating Ex- 
celence and Ethics for Success in Schools, Work and Beyond publicado bajo los auspicios de la Character Education Partnership y el Center for the 4th and 5th R's: Respect and Responsability $^{2}$. En esta investigación, basada en recientes teorías de la Psicología del Desarrollo, se retoman algunas de las ideas de Lickona propuestas en la década anterior.

Ya era constante en el pensamiento de Lickona la idea de que el objetivo de la EC es la educación en la virtud. Entendía el carácter como una disposición interior que permite a la persona responder bien ante situaciones morales, y considera que su educación debe tomar en cuenta los "componentes psicológicos del carácter" -conocer el bien, amar el bien y hacer el bien- con el objetivo de llevar al estudiante a la madurez moral. Su comprensión del carácter es entonces tripartita: mente, corazón y acción. La medida última del carácter es la acción. El autor proponía entonces metodologías específicas que buscaban el desarrollo de cada uno de estos elementos (Lickona, 1991; 1997, 1999).

Estas ideas son retomadas en su obra posterior y reformuladas. Lickona y Davidson (2005) sintetizan en el nuevo título de su modelo la idea esencial: las escuelas deben concentrarse en el cultivo de dos dimensiones: la inteligencia (en el sentido de inteligencia multidimensional) y la bondad (en el sentido multidimensional de madurez moral) y que ha sido misión central de la educación a lo largo de la historia.

En su propuesta hacen suya una cita del ex presidente estadounidense Franklin D. Roosevelt que dice que "educar la razón del hombre y no su moral, se convierte en educar una amenaza para la sociedad" (Lickona y Davidson, 2005). Pero la máxima contraria es igualmente negativa: educar a una persona moralmente y no en su inteligencia tendrá como resultado otra amenaza; será siempre perjudicial para la sociedad tener médicos, abogados, o mecánicos que sean honestos pero incompetentes en sus funciones.

Reconocen que la educación integral (mente, corazón y acción) debe abarcar tanto una dimensión moral como una dimensión "ejecutiva", promoviendo no sólo la formación de los pensamientos, sentimientos y acciones relacionados con el actuar moral, sino además el desa-

\footnotetext{
${ }^{2}$ Se tomó la decisión de denominar este apartado "el modelo de Thomas Lickona", pues aunque Davidson es coautor en el principal estudio que se reseña en este apartado ( Lickona y Davidson, 2005), se ha deseado complementar la información con bibliografía en la que solamente Lickona es autor. La figura de Lickona tiene renombre dentro del Movimiento, y se deseó resaltar su liderazgo en el título del apartado.
} 
rrollo de las capacidades prácticas para la excelencia personal en un sentido más amplio (Davidson, Lickona y Khmelkov, 2008; Lickona y Davidson 2005).

Para ellos una persona con carácter responde con excelencia a los retos cotidianos, sean de tipo moral o simplemente vinculados con sus actividades cotidianas. Plantean una visión bi-partita de carácter con:

a) una dimensión relacionada con la habilidades intelectuales, que denomina "carácter ejecutivo" - performance character-. Se relaciona con la educación de la inteligencia. Esta dimensión del carácter se orienta hacia la excelencia personal. Incluye aspectos como la diligencia, una sólida ética de trabajo, actitud positiva, autodisciplina y perseverancia, y es considerada por este autor como una "orientación hacia el auto-dominio" -self-mastery orientation-.

b) una dimensión vinculada al actuar moral denominada“carácter moral" - moral character-. Se relaciona con la educación moral. Se orienta a la educación del "carácter moral", dimensión que establece la forma en la que la persona se relaciona consigo mismo y con los demás. Incluye virtudes como la integridad, justicia, bondad y respeto, y tiene una orientación relacional. Este aspecto se refiere entonces al desarrollo de las cualidades necesarias para un comportamiento ético. Lickona y Davidson afirman que esta dimensión del carácter permite a la persona tratar a los demás -y a sí mismo- con respeto y cuidado actuando además con integridad ética.

Narvaez (2005) resalta cuatro contribuciones muy concretas de este modelo: 1) es un modelo integrativo que propone un "pensamiento correcto" (basado en el pensamiento platónico) integrado con un "comportamiento correcto" (basado en el pensamiento aristotélico), 2) el término "virtud" y su composición tripartita: conocimiento moral, sentimiento moral y comportamiento moral. 3) promueve la centralidad de la misión educativa en términos morales y 4) se nutre de aportaciones del ámbito psicoeducativo para fortalecer sus planteamientos (e.g. instrucción metacognitiva, aprendizaje cooperativo, motivación). Entre las críticas plantea dos carencias profundas del modelo: la falta de mayor rigor científico de su definición de carácter y de una pedagogía sistemática validada que de soporte a sus planteamientos. 
Narvaez es profesora de la Notre Dame University y directora del Center for Ethical Education and Development, vinculado a esta misma universidad. Su trabajo ha quedado plasmado en un modelo de EC que ha denominado Integrative Ethical Education (IEE). Dicho modelo se concretó en un proyecto educativo llamado Community Voices and Character Education (1998-2002), que se puso en marcha con buenos resultados en varios centros educativos del estado de Minnesota gracias a una financiación del Departamento de Educación de los EE UU (Lapsley y Narvaez, 2006).

La distinción medular entre el IEE y las otras propuestas, ya descritas en este trabajo, es que este modelo está basado en una nueva aproximación a las nociones de "carácter" y “educación”, lograda gracias a la integración de presupuestos de la EC, los modelos de educación moral cognitiva (especialmente de la propuesta kohlberiana) y algunas aportaciones del ámbito psicológico y educativo surgidas en las últimas décadas (Lapsley y Narvaez, 2006; Liddell, 2007; Narvaez 2002; 2007; 2008; Narvaez y Lapsley, 2005). Según la autora, el IEE:

a) Desea rescatar nociones de la filosofía clásica como eudonomia -"“florecimiento humano"-, arete -“excelencia”-, phronesis --“sabiduría práctica”- y tecne "expertice $o$ aprendizaje experto"-.

b) Tiene como punto de partida una noción de carácter que se relaciona con las aportaciones de las ciencias cognitivas, específicamente con teorías que explican el desarrollo del expertise -“aprendizaje experto”- (Bransford, Brown y Cocking, 1999; Ericsson y Smith, 1991; Sternberg, 1998); y el aprendizaje autorregulado (Torrano y González-Torres, 2004; Zimmerman, Bonner y Kovach, 2002)

c) Se nutre de los hallazgos de las Ciencias de la Prevención y la Psicología Positiva para determinar el grupo de habilidades que conforman el "buen carácter". Es compatible con la perspectiva del $P Y D$, pues su objetivo es la dotación de competencias que faciliten una respuesta asertiva a las demandas de la adultez.

d) Busca articular una propuesta de mejores prácticas para la educación moral, basada en el conocimiento acumulado por el Movimiento en su primera etapa y en algunas contribuciones sobre el desarrollo moral cognitivo. 
La tesis central del IEE se puede resumir en el siguiente esquema:

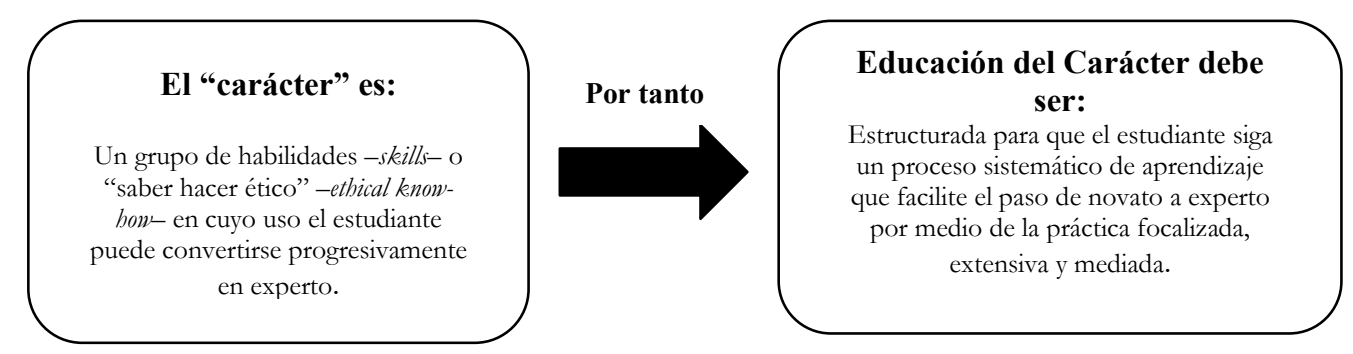

Figura 2. Tesis central del IEE. Inspirado en Narvaez, 2007.

Para explicar su modelo, la autora plantea las siguientes preguntas: ¿cómo aprenden los niños?; ¿en qué se diferencia entonces un novato de un experto?; ¿qué es eso que las personas con buen carácter conocen? Responde a estas cuestiones remitiéndose a las teorías actuales sobre cómo se aprende (constructivismo /mediación social), cómo se desarrolla el conocimiento experto y el papel de los procesos de autorregulación del aprendizaje, como hemos señalado.

Narvaez propone que lo que distingue a los expertos morales son 21 habilidades (p.e. comprender las expresiones emocionales, razonamiento ético, identificar criterios para enjuiciar, perseverar, planificar y tomar decisiones, etc.) muy desarrolladas que se pueden agrupar en 4 áreas (ver tabla 3):

Una aportación de esta autora-valiosísima para cualquier educador interesado en este tema- son las guías didácticas que propone para abordar la enseñanza de cada habilidad ${ }^{3}$.

El equipo dirigido por Darcia Narvaez se dedica actualmente a la búsqueda de estrategias para la medición de las habilidades morales que se trabajan en el modelo, al estudio de sus bases neurológicas (Narvaez, 2008) y de la identidad moral (Liddell, 2007) y se espera que el modelo siga enriqueciéndose con estas aportaciones. Quedan sobre el tapete algunos temas como la definición de la axiología de valores o de las virtudes a enseñar (en el Community Voices and Character Education era el propio centro educativo quien los decidía) y aportaciones más precisas sobre la relación, sostenida por la autora, entre la educación moral y el rendimiento académico.

\footnotetext{
${ }^{3}$ El lector podrá encontrar un ejemplo del material utilizado para el fortalecimiento de cada habilidad en la siguiente dirección electrónica: http://cee.nd.edu/curriculum/curriculum1.shtml. 
Tabla 3. El proceso modelo de la conducta ética. Inspirado en Narvaez, 2007

\begin{tabular}{|l|l|l|l|}
\hline $\begin{array}{c}\text { Sensibilidad Ética } \\
\text { (DARSE CUENTA) }\end{array}$ & \multicolumn{1}{|c|}{$\begin{array}{c}\text { Juicio Ético } \\
\text { (PENSAR) }\end{array}$} & \multicolumn{1}{c|}{$\begin{array}{c}\text { Foco Ético } \\
\text { (ENFOCAR) }\end{array}$} & \multicolumn{1}{c|}{$\begin{array}{c}\text { Conducta Ética } \\
\text { (ACTUAR) }\end{array}$} \\
\hline $\begin{array}{l}\text { Interpreta una situa- } \\
\text { ción moral tomando } \\
\text { en cuenta quién está } \\
\text { involucrado en ella, } \\
\text { qué acciones se deben } \\
\text { sibles acciones, y yas qué acción es } \\
\text { tomar, qué posibles } \\
\text { reacciones se pueden } \\
\text { dar y qué resultados } \\
\text { se pueden obtener. }\end{array}$ & & $\begin{array}{l}\text { Prioriza la acción } \\
\text { ética sobre otras me- } \\
\text { tas o necesidades. }\end{array}$ & $\begin{array}{l}\text { Pone en marcha la } \\
\text { acción ética, pues } \\
\text { sabe cómo hacerlo y } \\
\text { es capaz de vencer las } \\
\text { dificultades para con- } \\
\text { seguirlo. }\end{array}$ \\
\hline
\end{tabular}

\section{Conclusiones}

El Movimiento de Revitalización de la Educación del Carácter (MRC) es una iniciativa para la educación moral que pretende rescatar los principios aristotélicos de la educación para la virtud. La educación y psicología moral durante mucho tiempo ha estado dominada por las ideas del Desarrollo Moral Cognitivo propuesto por Kohlberg. El MRC, frente a ese hegemonismo, aunque integrando sus aportaciones, presenta propuestas para la EC que se han ido nutriendo también de teorías e investigaciones consistentes de distintos ámbitos científicos con resultados positivos.

Este movimiento surgió en EE UU en los años 90 del S. XX en un contexto de preocupación por los crecientes problemas de muchos niños y jóvenes a los que la educación moral imperante no parecían responder y también en un contexto favorecedor. La historia de la educación moral en este país tiene hitos particulares que influyeron tanto en el declive de la EC como en el posterior crecimiento del MRC. Resulta claro que en ese proceso ha sido vital el trabajo conjunto entre el gobierno, ONGs, centros de investigación y centros educativos lo que explica en gran parte su rápida difusión en los EE UU. Sin duda sus aportaciones son un importante punto de referencia para la implantación de propuestas de EC en otros contextos que enfrentan problemáticas en relación a la juventud cada vez más similares a la estadounidense.

Hemos detallado algunas de sus fortalezas y debilidades y cómo el MRC se ha enriquecido y se está consolidando teórica y empíricamente con las aportaciones que ha traído el 
cambio de perspectiva en el ámbito de la Prevención (PYD) y la Psicología Positiva, lo que ha alimentado el interés científico y práctico por la EC. Podemos destacar dos interesantes contribuciones:

a) La educación del carácter es preventiva: Esas perspectivas han apoyado los planteamientos del MRC al centrar su mirada en las capacidades y posibilidades de los educandos para el actuar moral y para el pleno desarrollo de los jóvenes.

b) Reaparición de las nociones de virtud y carácter en el ámbito psicoeducativo: La Psicología Positiva hace una aportación importantísima al rescatar la noción de virtud y carácter como centro de estudio científico. Propone que se puede hacer un estudio sistemático de las virtudes, de las fortalezas de carácter y "la vida buena" y ofrece una primera obre en este sentido: el Character Strenghts and Virtues: A Handbook and Classification de C. Peterson y M. Seligman.

Respecto a recientes aportaciones del Movimiento, consideramos especialmente valiosa la de Navaez. Su modelo (IEE) y sus planteamientos punteros acerca de la EC han sembrado el interés por este tema dentro de la Psicología de la Educación, puesto que se apoya en perspectivas sociocognitivas, constructivistas y neurobiológicas y, concretamente, en las investigaciones actuales sobre el desarrollo del conocimiento experto (expertice). Con este marco plantea una nueva comprensión de la noción del carácter, las dimensiones a trabajar y cómo hacerlo. Además, su modelo se ha llevado a la práctica en el programa Community Voices and Character Education que está siendo validado empíricamente con buenos resultados.

Como conclusión final consideramos que se puede ver la EC -virtudes- como un camino válido para el desarrollo positivo de todos los jóvenes y en consecuencia para la prevención de problemas. Diversas teorías psicológicas respaldan esta premisa, y los resultados obtenidos por el MRC hasta la fecha son prometedores. De cara al futuro todavía es necesario salvar diversos obstáculos como: los problemas de lenguaje, la definición de los constructos e instrumentación, una mayor integración de las diversas perspectivas que se recogen bajo la rúbrica de $\mathrm{EC}$, validación empírica de los programas de intervención y más desarrollo de estudios longitudinales (Berkowitz et al., 2008).

Hemos querido ofrecer al lector una síntesis de las información relevante sobre el pasado, presente y futuro del MRC en los EE UU. Una cuestión candente que queremos dejar planteada es hasta qué punto se pueden incorporar las propuestas de este Movimiento en paí- 
ses con una historia y contexto actual diferente del estadounidense. Las autoras valoramos especialmente la riqueza teórica y metodológica de las propuestas del MRC, y consideramos que pueden ser una gran aportación para los trabajos que, en esta línea, se están haciendo fuera del contexto estadounidense $\mathrm{y}$, concretamente, en nuestro país aunque no se encuadren bajo la denominación de educación del carácter.

\section{Referencias}

Abundant Assets Aliance (2002) Home. Extraído el 25 de febrero del 2008 de http://www.abundantassets.org/

Altarejos, F. y Naval, C. (2000) Filosofía de la educación. Pamplona: EUNSA.

Aristóteles (1994). Etica a Nicómaco. Madrid: Centro de Estudios Constitucionales.

Baumeister, R., Boden, J., y Smart, L. (1996) Relation of Threatened Egotism to Violence and Agression: The Dark Side of High Self-Esteem. Psychological Review, 103, 5-33.

Baumeister, R., Campbell, J., Krueger, J. y Vohs, K. (2005) Exploring the Self-Esteem Myth. Scientific American Mind, 1(292), 84-91.

Basttistich, V. (2005) Character Education, Prevention and Positive Youth Development. Washington, DC: Character Education Partnership. Extraído el 24 de febrero del 2001 de www.communityofcaring.org/servicesandreasources/

Bransford,J.D., Brown, A.L., y Cocking, R.R. (Eds) (1999). How people learn: Brain, mind, experience and school. Washington, DC: National Academy Press

Bennigna, J. (Ed.) (1991) Moral, Character, and Civic Education. New York, NY: Teacher College Press.

Bennigna, J. y Wynne, E. (1998). Keeping in character: A time-tested solution. Phi Delta Kappan, 6 (79), 439-447.

Benson, P., Scales, P., Leffert, N. y Roehlkepartain, E. (1999) A fragile foundation: the State of Developmental Assets among American Youth. Minneapolis, MN: Search Institute.

Benson, P. y Pittman, K. (2001). Trends in Youth Development: Visions, Realities and Challenges. Norwell, MA: Kluwer Academic Publishers.

Benson, P. y Saito, R. (2001). The Scientific Foundations of Youth Development. En P. Benson, y K. Pittman (2001) (Eds.) Trends in Youth Development: Visions, Realities and Challenges (pp.135-153). Norwell, MA: Kluwer Academic Publishers.

Battistich, V. (2005). Character Education, Prevention and Positive Youth Development. Washington, DC: Character Education Partnership. 
Berkowitz, M. (1995). Educar a la persona moral en su totalidad. Revista Iberoamericana de Educación, 1, 1-25. Extraído el 25 de abril del 2007 de www.ricoei.org/rie09903.html Berkowitz, M. (1997). Integrating Structure and Content in Moral Education. Ponencia presentada en la Jornada Developmental Perspectives to Character Education. Chicago, IL. Extraído el 25 de abril del 2007 de www.tigger.uic.edu

Berkowitz, M. (1998). The education of the complete moral person. Extraído el 25 de abril del 2007 de www.tigger.uic.edu

Berkowitz, M. (1999). Buiding a Good Person. En M. Williams, E. Schaps (Eds.), Character Education: The Foundation for Teacher Education. (pp. 19-23) Washington, DC: Character Education Partnership.

Berkowitz, M. (2000). Character Education as Prevention. En W.B. Hansen, S.M Giles, M.D. Fernow-Keney (Eds.), Increasing drug prevention effectiveness: Readings for educators (pp.43-63). Clemmons, NC: Tanglewood Research.

Berkowitz, M. (2002). The science of character education. En W. Damon (Ed.), Bringing in a New Era in Character Education. (pp. 43-63) Stanford, CA: Hoover Press Publication. Berkowitz, M. y Bier, M. (2004). Research Based Character Education. The Annals of the American Academy of Political and Social Science, 591, 72-84.

Berkowitz, M. y Bier, M. (2005a). What works on character education: A research-driven guide for educators. St. Luis, MI: Character Education Partnership- John Templeton Foundation.

Berkowitz, M. y Bier, M. (2005b). What works on character education: A report for policy makers and opinion leaders. St. Luis, MI: Character Education Partnership- John Templeton Foundation.

Berkowitz, M., Sherblom, S., Bien, M. y Battictich, V. (2005). Educating for Positive Youth Development. En M. Killien, Handbook of Moral Development (pp.683-701), Mahwah, NJ: Lawrence Erlbaum Associates.

Berkowitz, M.W.; Battistich, V.A.; Bier, M. (2008). What Works in Character Education: What is Known and what need to be Known. En L.P. Nucci y D. Narvaez . Handbook of Moral and Character Education (pp. 414-431) New York: Rouledge.

Bernal, A. (1998). Educación del carácter, educación moral: propuestas educativas de Aristóteles y Rousseau. Pamplona: EUNSA.

Bernard, B. (2004). Resiliency: What we have learned. San Francisco, CA: WestEd. 
Bonnano, G.A. (2004). Loss, Trauma and Human Resilience: Have we underestimated the Human Capacity to Thrive After Extremely Aversive Events? American Psychologist, 59(1), 20-28.

Brown, J., D’Emidio-Caston, M., y Bernard, B. (2001). Resilience Education. Thousand Oaks, CA: Corwin Press.

Catalano, R., Berlung, L., Ryan, J., Lonczak, H. y Hawkins, D. (2004). Positive Development in the United States: Research Findings and Evaluations of Positive Youth Development Programs. The Annals of the American Academy of Political and Social Science, $591,98-123$

Catalano, R.F.; Hawkins, J.D.; Toumbourou, J.W. (2008). Positive Youth Development in the United States: History, Efficacy and Links to Moral and Character Education. En L.P. Nucci y D. Narvaez. Handbook of Moral and Character Education (pp. 459-483) New York: Rouledge.

Center for the Advancement of Ethics and Character (2006). Character Education Manifesto. Extraído 08/02/2007 de http://www.bu.edu/education/caec/files/manifesto.htm

Center for the 4th and 5th Rs: Respect and Responsibility. (2006). About us. Extraído el 13/04/2007 de http://www.cortland.edu/character/default.asp

Character Education Partnership (CEP) (1999). National Schools of Character: Best Practices and New Perspectives. Washington, DC: Autor.

Character Education Partnership (CEP) (2005). About us. Extraído el 3 de noviembre, 2007 http://www.character.org/site

Character Education Partnership (CEP) (2006). Character Education Quality Standarts: A self-Assesment Tool for Schools and Districts. Washington, DC: Autor.

Character Education Partnership (CEP) (2009). Letter to President Elect Obama. Extraído de http://www.character.org/lettertoobama el 22 de febrero, 2009.

Character Counts! (2007a). The Aspen Declaration. Extraído el 30 de enero del 2008 de http://charactercounts.org/aspen.htm

Character Counts! (2007b). About us. Extraído el 30 de enero del 2008 de http://charactercounts.org/aspen.htm

Chafouleas, S. y Bray, M. (2003) Introducing Positive Psychology: Finding a Place Within School Psychology. Psychology in the Schools, 4(1), 1-5.

Clary, G. y Rhodes, J. (2006). Mobilizing Adults for Positive Youth Development. New York, NY: Springer Science. 
Cunningham, C. (2005). A Certain and Reasoned Art: The rise and Fall of Character Education in America. En D. Lapsley; C. Power (Eds.), Character Psychology and Character Education (pp.166-200). Notre Dame, IN: Notre Dame Press.

Csikszentmihalyi, M. y Csikszentmihalyi, I. (Eds.) (2006). A life worth living. New York, NY: Oxford Press.

Damon, W. (1995). Great expectations: Overcoming the culture of indulgence in America's homes and schools. New York, NY: The Free Press.

Damon, W. (2002). Bringing in a new era in character education. Stanford, CA: Hoover Press Publication.

Damon, W. (2008). The path to purpose. Helping our Children Find Their Calling in Life. New York: Free Press

Davidson, M., Lickona, T., y Khmelkov, V. (2008). Smart \& Good Schools: A New Paradigm for High School Character Education. En L.P. Nucci y D. Narvaez. Handbook of Moral and Character Education (pp. 370-391). New York: Rouledge.

Devine, T., Seuk, J. y Wilson, A. (Eds.) (2000). Cultivating Heart and Character. Chapel Hill, NC: Character Development Publishing.

De la Fuente, J., Peralta, F.J. y Sánchez, M.D. (2009) Autorregulación personal y percepción de los comportamientos escolares desadaptativos. Psicothema, 21 (4), 548-554

DeRoche, E. y Williams, M. (1998). Educating hearts and minds. California, CA: Corwin Press.

Elzo, J. (2006). Los jovenes y la felicidad: ¿Dónde la buscan? ¿Dónde la encuentran? Madrid: PPC.

Ericsson, K.A. y Smith, J. (1991). Toward a General Theory of Expertise. New York: Cambridge University Press.

Fernández-Berrocal, P. y Aranda, D. (2008). La inteligencia emocional en la educación. Electronic Journal of Research in Educational Psychology, 15, 6 (2), 421-436

Gimeno Sacristán, J. (2005). La educación que aún es posible. Madrid: Morata.

Glantz, M. y Johnson, J. (1999) (Eds.) Resilience and Development: Positive Life Adaptations. New York, NY: Kluwer Academic/Plenum Publishers.

González-Torres, M.C. (2001). Las concepciones psicológicas del yo en la postmodernidad: implicaciones para la educación moral y cívica. En C. Naval y C. Urpi (Eds.), Una voz diferente en educación moral. (pp.41-78) Pamplona: EUNSA. 
González-Torres, M. C. (2003). Familia y autoestima de los hijo (nº 5) En VVAA. Educación para la prevención de las drogodependencias. Material para la formación de padres curso 2002-2003. Madrid: Fundación Proforpa.

Henderson, N., Bernard, B. y Sharp-Light, N. (1999). Resiliency in action: Practical Ideas for Overcoming Risks and Building Strenghts in Youth, Families and Communities. San Diego, CA: Resiliency in Action, INC.

Hoff, C. (2002). The science of character education. En W. Damon (Ed.), Bringing in a new era in character education. (pp. 43-63) Stanford, CA: Hoover Press Publication.

Iriarte, C., Alonso-Gancedo, N. y Sobrino, A. (2006). Relationships between Emocional and Moral Development to be taken into account in the Educational Setting. Proposed Intervention Program. Electronic Journal of Research in Educational Psychology, 4(1), $177-212$

Josephson Institute (2001). La declaración de Aspen. Extraído el 8 de febrero de http://www.charactercounts.org/Espanol/aspen.htm

Keyes, C. y Haidt, J. (2003). Flourishing: Positive Psychology and the life well lived. Washington, DC: American Psychological Association.

Lapsley, D. y Narvaez, D. (2004). A Social-Cognitive Approach to the Moral Personality. En D. Lapsley; D. Narvaez (Eds.), Moral Development, Self and Identity (pp.189-212). Mahwah, NJ: Lawrence Erlbaum Associates.

Lapsley, D. y Narvaez, D. (2005). Moral Psychology at the Crossroads. En D. Lapsley y C. Power (Eds.), Character Psychology and Character Education (pp.18-35). Notre Dame, IN: Notre Dame Press.

Lapsley, D. y Narvaez, D. (2006). Character Education. En W. Damon y R. Lerner (Eds.), Handbook of Child Psychology (pp.248-296). Hoboken, NJ: Wiley.

Leming, J. (1997). Research and Practice in Character Education: A Historical Perspective. En Molinar, A. (Ed.) The Construction of Childrens Character (pp. 31-44). Chicago, IL: The National Society for the Study of Education.

Lickona, T. (1991). Educating for Character. New York, NY: Bantam books.

Lickona, T. (1997). Educating for Character: A Comprehensive Approach. En Molinar, A. (Ed.) The Construction of Childrens Character (pp. 45-62). Chicago, IL: The National Society for the Study of Education.

Lickona, T. (1999). Educación del Carácter: Cultivar la Virtud. En C. Reigeluth (Ed.) Diseño de la instrucción. Teorías y modelos: Un nuevo paradigma de la teoría de la instrucción (pp. 139-160). Madrid: Aula XXI/Santillana. 
Lickona, T. y Davidson, M. (2005). Smart and Good High Schools: Integrating Excellence and Ethics for Success in School, Work and Beyond. Cortland, NY: Center for the 4th and 5th Rs: Respect and Responsibility/Washington, DC: Character Education Partnership.

Liddell, D. (2007). Ethical Identity and Moral-Related Variables: Spotlight on Darcia Narvaez, Tonia Bock, and Anna Gomberg. Journal of College Education, 1(XI), 1-3.

Linley, A. y Stephen, J. (Eds) (2004). Positive Psychology en Practice. Hoboken, NJ: John Wiley and Sons.

Linley, A., Stephen, J., Harrington, S. y Wood, A. (2006). Positive Psychology: Past, Present and (possible) Future. The Journal of Positive Psychology, 1(1), 3-16.

Lopez, S., Snyder, C.R. y Rasmussen, H. (2003). Striking a Balance: Developing a Complementary Focus on Human Weakness and Strength Through Positive Psychological Assessment. En S. Lopez, C.H. Snyder (Eds.), Positive Psychological Assessment (pp.320). Washington, DC: American Psychological Association.

López de Dicastillo, N., Iriarte, C., y González-Torres, M.C. (2008). Competencia social y educación cívica. Madrid: Síntesis.

Maddi, S. (2006). Building an Integrated Positive Psychology. The Journal of Positive Psychology, 1(4), 226-229.

Masten, A. (1999). Resilience Comes to Age: Reflections and the Past and Outlook for the Next Generation of Research. En M. Glantz y J. Johnson (Eds.), Resilience and Development: Positive Life Adaptations (281-296). New York, NY: Kluwer Academic/Plenum Publishers.

Masten, A. (2001). Ordinary Magic: Resilience Processes in Development. American Psychologist, 56(3), 227-238.

Masten, A. y Coatsworth, J.D. (1998). The Development of Competence in Favourable and Unfavourable Environments, American Psychologist, pp. 212-216.

Masten, A. y Reed, M (2002). Resilience in Development. En C.R. Snyder y S. Lopez (Eds.) Handbook of Positive Psychology (74-88).NY: Oxford University Press.

Mayerson Foundation (2007). Who we are. Extraído el 4 de marzo del 2008 de http://www.mayersonfoundation.org/whoweare/main.asp

McCain, J., y Salter, M. (2005). Character Is Destiny: Inspiring Stories Every Young Person Should Know and Every Adult Should Remember. NY: Random House. 
McClellan, E. (1992). Schools and the Shaping of Character: Moral Education in America, 1607-Present. Bloomington, IN: ERICK Clearinghouse for Social Science Education and the Social Studies Development Center.

McClellan, E. (1999). Moral Education in America: Schools and the Shaping of Character from Colonial Times to the Present. New York, NY: Teachers College Press.

Morrison, G., Brown, M., D'Incau, B., O’Farrell, S.T. y Furlong, M. (2006). Understanding Resilience in Educational Trajectories: Implications for Protective Possibilities. Psychology in the Schools, 1(43), 19-31.

Murphy, M. (2001). Three essential components of character development. En C. Naval y C. Urpi (Eds.), Una voz diferente en educación moral. (pp.111-129) Pamplona: EUNSA.

Murphy, M. (2002). Character Education in America's Blue Robbon Schools: Best Practices for Meeting the Challenge. Lanham, MA: The Scarecrow Press.

Munist, M., Santos, H., Kotliarenco, M.A., Suárez, E., Infante, F. y Grotberg, E. (1998). Manual de identificación y promoción de la resiliencia en niños y adolescentes. Washington DC: Organización Panamericana de la Salud/OMS. Extraído el 16 de abril del 2008 de http://resilnet.uiuc.edu/library/resilman/resilman.html

Nash, R. (1997). Answering the virtuecrafts. New York, NY: Teachers College Press.

Naval, C. (2001). What is character or moral education all about? En C. Naval y C. Urpi (Eds.), Una voz diferente en educación moral. (pp.131-146) Pamplona: EUNSA.

Narvaez, D. (2002). The Expertise of Moral Character. Ponencia presentada en The White House Conference on Character, Washington, DC. Extraído el 16 de febrero del 2007 de www.whitehouse.gov/

Narvaez, D. (2005). Integrative Ethical Education. En M. Killen y J. Smetana (Eds.) Handbook of Moral Development (pp.703-734). Mahwah, NJ: Lawrence Erlbaum.

Narvaez, D. (2007). Integrative Ethical Education: Putting Flourishing back to Character Education. Extraído el 5 de octubre del 2007 de http://www.nd.edu/ dnarvaez/NarvaezISPP.pdf

Narvaez, D. y Lapsley, D. (2005). The psychological Foundations of Everyday Morality and Moral Expertise. En D. Lapsley; C. Power (Eds.), Character Psychology and Character Education (pp.140-165). Notre Dame, IN: Notre Dame Press.

Narvaez, D. (2008). Human Flourishing and Moral Development: Cognitive and Neurobiological perspectives of Virtue Development. En L.P. Nucci y D. Narvaez . Handbook of Moral and Character Education (pp. 310-327). New York: Rouledge 
Nucci, L. (2005). Education for Moral Development. En M. Killen y J. Smetana (Eds.) Handbook of Moral Development (pp. 657-681). Mahwah, NJ: Lawrence Erlbaum Associates.

Nucci, L.P. y Narvaez, D. ( 2008) (Eds.) Handbook of Moral and Character Education. New York: Routledge.

Palmour, J. (1986). On moral character: A practical guide to Aristotle's virtues and vices. Washington, DC: The Archon Institute for Leadership Development.

Papalia, D., Wendkos, S. y Duskin, R. (2001) Desarrollo Humano. Bogotá: McGraw-Hill Interamericana S.A.

Park, N. (2004) Character Strenghts and Positive Youth Development. The Annals of the American Academy of Political and Social Science, 591, 40-54.

Park, N., Peterson, C. y Seligman, M. (2006) Character Strenghts in fifty-four nations and the fifty US States. The Journal of Positive Psychology, 2(1), 118-129.

Park, N., y Peterson, Ch. (2009). Character Strengths: Research and Practice. Journal of College \& Character, 10(4), 1-10

Pérez, P.M. y Cánovas, P. (2002). Valores y pautas de interacción familiar en la adolescencia. Madrid: Fundación Santa María.

Peterson, C. (2006a). A Primer in Positive Psychology. NY: Oxford Press.

Peterson, C. (2006b). The Values un Action (VIA) Classification of Strenghts. En M. Csikszentmihalyi y I. Csikszentmihalyi (Eds.), A life worth living (pp.29-48). New York, NY: Oxford Press.

Peterson, C. y Seligman, M. (2004). Character Strenghts and Virtues: A Handbook and Classification. Washington, DC: American Psychological Association/New York, NY: Oxford University Press.

Pittman, K., Irby, M. y Ferber, T. (1996) Unfinished Business: Further Reflexions on a Decade of Promoting Youth Development. Maryland, M.D: IYF-US, International Youth Foundations.

Pittman, K. (2000). Balancing the Equation; Communities Supporting Youth, Youth Supporting Communities. CDY Journal, 1(1), 1-8.

Pittman, K., Irby, M., Tolman, J., Yohalem, N. y Ferber, T. (2001). Preventing Problems, Promoting Development, Encouraging Engagement: Competing Priorities or Inseparable Goals? Maryland, M.D: IYF-US, International Youth Foundations. 
Power, C. (1997). Understanding the Character in Character Education. Ponencia presentada en Developmental Perspectives and Approaches to Character Education. Chicago, Il. Extraído el 27 de febrero del 2007 de www.tigger.uic.edu/

Quintana, C. (1995). Pedagogía Moral: El desarrollo moral integral. Madrid: Dykinson.

Roberts, M., Brown, K., Johnson, R. y Reinke, J. (2002). Positive Psychology for Children: Development, Prevention and Promotion. En C.R. Snyder y S. López (Eds.) Handbook of Positive Psychology (663-675). New York, NY: Oxford University Press.

Rodríguez, M.C., Muñoz, M.V., Pérez, P. y Sánchez, I. (2005). Los adolescentes españoles y su salud: Un Análisis en chicos y chicas de 11 a 17 años. Madrid: Ministerio de Sanidad y Consumo. Extraído el 9 de marzo del 2007 de http://www.msc.es/

Rolf, J. y Glantz, M. (1999). Resilience: An interview with Norman Garmezy. En M. Glantz y J. Johnson (Eds.), Resilience and Development: Positive Life Adaptations (5-14). New York, NY: Kluwer Academic/Plenum Publishers.

Roth, J. y Brooks-Gunn, J. (2003). Youth Development Programs: Risk, Prevention and Policy. Jounal of Adolescent Health, 32, 170-182.

Ryan, K. (1999). Character Education Paper. En M. Williams y E. Schaps. Character Education: The Foundation of Teachers Education. Washington, DC: Character Education Partnership.

Ryan, K. y Bohlin, K. (1999) Building Character in Schools: Practical Ways to Bring Instruction to Life. San Francisco, CA: Jossey-Bass Publishers.

Ryan, K. y Lickona, T. (1991) (Eds.). Character Development in Schools and Beyond Washington, DC: The Council for Research in Values and Philosophy.

Search Institute (2007). What's new. Extraído el 25 de febrero del 2008 de http://www.searchinstitute.org/whatsnew/

Scales, P., Foster, K., Mannes, M., Horst, M., Pinto, K. y Rutherford, A. (2005) SchoolBusiness Partnerships, Developmental Assets, and Positive Outcomes Among Urban High School Students: A Mixed-Methods Study. Urban Education, 40(2), 144-189.

Schaps, E., Battistich, V. y Solomon, D. (1997) School as a Caring Community: A Key to Character Education. En A. Molinar (Ed.) The Construction of Childrens Character (pp. 127-139). Chicago, IL: The National Society for the Study of Education.

Seligman, M. (1996). The Optimistic Child: A Proven Program to Safeguard Children Against Depression and Build Life-Long Resilience. New York, N.Y.: Harper Perenial. 
Seligman, M. (2000). Positive Psychology. En J. Gillham (Ed.), The Science of Optimism and Hope: Research Essays in Honor of Martin Seligman (pp.415-429). Radnor, PA: Templeton Foundation Press.

Seligman, M. (2003). La Auténtica Felicidad. Barcelona: Ediciones B.

Seligman, M. y Csikszentmihalyi, M. (2000). Positive Psychology: An Introduction. American Psychologyst, 55(1), 5-14.

Seligman, M., Steen, T., Park, N. y Peterson, C. (2005). Positive Psychology Progress: Empirical Validation of Interventions. American Psychologist, 60(5), 410-421.

Sheldon, K. y King, L. (2001) Why Positive Psychology is Necessary. American Psychologist, 56(3), 216-217.

Small, S. y Memmo, M. (2004) Contemporary Models of Youth Development and Problem Prevention: Toward an Integration of Terms, Concepts and Models. Family Relations, 53(1), 3-11.

Smith, L. L., y Elliott, C. H. (2001). Hollow kids: Recapturing the Soul of a Generation lost to the Self-Esteem Myth. Rocklin, CA: Prima.

Snyder, C.R. y López, S. (2002). Handbook of Positive Psychology. New York, NY: Oxford University Press.

Steen, T., Kachorek, L. y Peterson, C. (2003). Character Strenghts Among Youth. Journal of Youth and Adolescence, 32(1), 5-16.

Sternberg, R. (1998). Abilities are forms of developing expertise, Educational Researcher, 3, 22-35.

Trianes, M.V. y Fernández, C. (2001). Aprender a ser personas y a convivir: Un programa para secundaria. Bilbao: Desclee de Brower.

Twenge, J.M. (2006). Generation Me: Why Today's Young Americans Are More Confident, Assertive, Entitled - and More Miserable than Ever Before. New York: Free Press.

Twenge, J.M. y Campbell, W.K. (2009). The narcissism Epidemic: Living in the Age of Entitlement. New York: Free Press

Torrano, F. y Gonzalez-Torres, M.C. (2004). Self Regulated Learning: Current and Future Directions. Electronic Journal of Research in Educational Psychology, 2(1), 1-34.

Urra, J. (2006). El pequeño dictador: Cuando los padres son las victimas. Madrid: La Esfera de los Libros.

Vera, B. (2006). Psicología Positiva: Una nueva forma de entender la psicología. Papeles del Psicólogo, 1(27), 1-5. 
Vera, B. (2008). Cuestionario VIA de fotalezas de personales (VIA Signature Cuestionary Strenghts). Extraído el 6 de marzo del 2008 de www.psicologiapositiva.com/via/html

VIA Institute of Character (2007). History. Extraído el 3 de marzo 2008 de www.viastrenghts.org

Werner, E., (1996). Vulnerable but invincible: High risk children from birth to adulthood. European Child \& Adolescent Psychiatry, 5, 47-55.

Weisberg, R., Kumpfer, K. y Seligman, M. (2003). Prevention that works for children and youth: An Introduction. American Psychologist, 58, 425-432.

Williams, D., Yanchar, S., Jensen, L. y Cherryl, L. (2003). Character Education in a Public High School: a multi-year inquiry into Unified Studies. Journal of Moral Education, 1(32), 3-33.

Wynne, E. y Hess, M. (1991). Trends in American Youth Character Development. En K. Ryan, T. Lickona (Eds.), Character Development in Schools and Beyond (pp.29-48). Washington, DC: The Council for Research in Values and Philosophy.

Wynne, E. y Ryan, E. (1997). Reclaiming our schools: Teaching character, academics and discipline. Bergen County, NJ: Prentice-Hall.

Zimmerman, B.J.; Bonner, S. y Kovach, R. (2002). Developing self-regulated learners. Washington, D.C.: American Psychological Association 